Proceedings of the XXXVIII International School and Conference on the Physics of Semiconductors "Jaszowiec" 2009

\title{
Spin-Dependent Transient Effects in Electron Transport in the Quantum Dot System
}

\begin{abstract}
P. PARAFInIUK AND R. TARANKO*
Institute of Physics, M. Curie-Skłodowska University, pl. M. Curie-Skłodowskiej 1, 20-031 Lublin, Poland

We study the effects of the Coulomb interaction $U$ between the electrons of opposite spins localized in the quantum dot on the time-dependent electron transport through the quantum dot coupled with two non-magnetic leads. We calculate the transient spin-dependent current generated by a pulsed bias voltage applied across the quantum dot system via the equation of motion method for appropriate correlation functions. The influence of the Coulomb interaction on the quantum coherent oscillations and beats of the current, spin current and current polarization ratio is studied. Our results indicate that the coherent beats in both the total and spin currents observed in the case of vanishing Coulomb interaction are strongly suppressed by non-zero Coulomb interaction. We also show that in the case of the pulsed bias almost fully polarized current occurs in some time windows for non-vanishing Coulomb interaction contrary to almost pure charge or spin current at vanishing $U$.
\end{abstract}

PACS numbers: 72.25.-b, 73.63.Kv, 85.75.-d

\section{Introduction}

The spin coherent dynamics and the electron transport through the quantum dot (QD) have mostly been studied beyond a transient timescale after a bias voltage was turned on (e.g. [1-4]). However, in the context of emerging field of spintronic or recent experimental advances in the studies of the QD subjected to ultrafast voltage pulses, it would be desirable to perform the time-dependent simulations of the charge and spin dynamics also in a transient timescale when some QD system parameters were suddenly changed. The calculations in which no Coulomb interaction between the electrons localized on QD was accounted for were reported in literature (see, e.g. [5-7]). The coherent quantum oscillations and beats of the total current and spin current flowing through the QD coupled with two leads were obtained for the QD placed in some external magnetic field. On the other hand, similar calculations performed for the magnetic QD with the intra-dot spin-flip scattering taken into consideration show that the amplitude of the beats is significantly suppressed [8]. As the intra-dot Coulomb interaction is much more important in describing the properties of the electron transport through the QD in comparison with the intra-dot spin-flip scattering, first of all, it would be very desirable to consider its influence on the total current and spin current. In this communication the results of such studies are reported.

\section{Theory}

We consider the QD coupled with two non-magnetic leads described by the Hamiltonian $H=H_{\text {Dot }}+H_{\mathrm{L}}+$

\footnotetext{
* corresponding author; e-mail: taranko@kft.umcs.lublin.pl
}

$H_{\mathrm{R}}+H_{\mathrm{T}}$. Here $H_{\mathrm{L} / \mathrm{R}}=\sum_{k \sigma} \epsilon_{k \sigma}(t) c_{k \sigma}^{+} c_{k \sigma}$, where $c_{k \sigma}$ $\left(c_{k \sigma}^{+}\right)$annihilates (creates) an electron with the wave vector $k$ and spin $\sigma$ in the left (L) or right (R) lead and $\epsilon_{k \sigma}(t)$ is the energy of lead electrons, $H_{\text {Dot }}=\sum_{\sigma}\left[\epsilon_{d \sigma}(t)+\right.$ $\left.\sigma E_{\mathrm{Z}} / 2\right] c_{d \sigma}^{+} c_{d \sigma}+U c_{d \uparrow}^{+} c_{d \uparrow} c_{d \downarrow}^{+} c_{d \downarrow}$, where $\epsilon_{d \sigma}(t)$ is the QD level, $E_{\mathrm{Z}}$ is the Zeeman energy due to some external field, $U$ is the Coulomb interaction between two electrons localized on the $\mathrm{QD}, c_{d \sigma}\left(c_{d \sigma}^{+}\right)$annihilates (creates) an electron in the QD and $H_{\mathrm{T}}=\sum_{\alpha k \sigma} V_{\alpha k \sigma} c_{k \sigma}^{+} c_{d \sigma}+$ H.c., where $\alpha=\mathrm{L}, \mathrm{R}$ and $V_{\alpha k \sigma}$ is the coupling parameter. The current from the $\alpha=\mathrm{L}$, R lead to the QD is calculated from the time evolution of the total number operator of the electrons in the $\alpha$-th lead and for example for $\alpha=\mathrm{L}$, one obtains

$$
j_{\mathrm{L}}(t)=\frac{2 e}{\hbar} \operatorname{Im} \sum_{k \sigma} V_{k \sigma}(t)\left\langle c_{d \sigma}^{+}(t) c_{k \sigma}(t)\right\rangle,
$$

where the operators are the Heisenberg ones and the averages are with respect to the equilibrium grand-canonical ensemble. The populations of the QD energy levels are obtained from the knowledge of the correlation function $\left\langle c_{d \sigma}^{+}(t) c_{d \sigma}(t)\right\rangle$. The required functions $\left\langle c_{d \sigma}^{+}(t) c_{k \sigma}(t)\right\rangle$ and $\left\langle c_{d \sigma}^{+}(t) c_{d \sigma}(t)\right\rangle$ can be obtained writing down the equation of motion (EOM) for them. As the Coulomb interaction $U$ is included in our model, the set of equations produced by the EOM method for higher order correlation functions cannot be closed without applying an approximate truncation procedure. In our case we have decoupled the appropriate higher-order correlation functions on a sufficiently deep level of calculations in order to be sure that the electron correlation effects are included beyond the mean field approximation.

\section{Results and discussion}

In the following we have calculated the spin resolved currents and $\mathrm{QD}$ occupations in a transient timescale 
when the bias voltage was suddenly applied to the QD system in question in the form of one step-like pulse or repeated step-like pulses with the period $T$. In the numerical calculations we have chosen $e=\hbar=1$ units, all energies are measured in $\Gamma_{\mathrm{L}}^{\sigma}=\Gamma_{\mathrm{R}}^{\sigma}=\Gamma$ units, the time and current in $\hbar / \Gamma$ and $e \Gamma / \hbar$ units, respectively. Here $\Gamma_{\mathrm{L}}^{\sigma}=\sum_{k}\left|V_{k \sigma}\right|^{2} \delta\left(\epsilon-\epsilon_{k \sigma}\right)$. We assume that for zero bias voltage the empty QD energy levels $\epsilon_{\uparrow}$ and $\epsilon_{\downarrow}$ are below and above the Fermi energy of both leads. At $t=0$ the tunneling between QD and leads is switched on, thus resulting in the subsequent current flowing from the leads to the QD until the equilibrium conditions are achieved. Now the step-like external bias is switched on resulting in sudden shift of QD energy levels and the right lead Fermi level.

In Fig. 1 we show the resulting spin current $j^{\downarrow}-j^{\uparrow}$ and the total current $j^{\uparrow}+j^{\downarrow}$ calculated for the parameters which allow us to observe well defined structures in the transient regime. For vanishing Coulomb interaction we observe typical quantum beats and coherent oscillations of the total and spin currents $[5,7]$. The quantum beats are the results of the slightly different frequencies of the spin-up and spin-down currents [5]. The situation is quite different for non-zero Coulomb interaction. Now the frequencies of the transient spin-up and spin-down currents differ much more in comparison with the $U=0$ case and the amplitude of the quantum beats is suppressed.

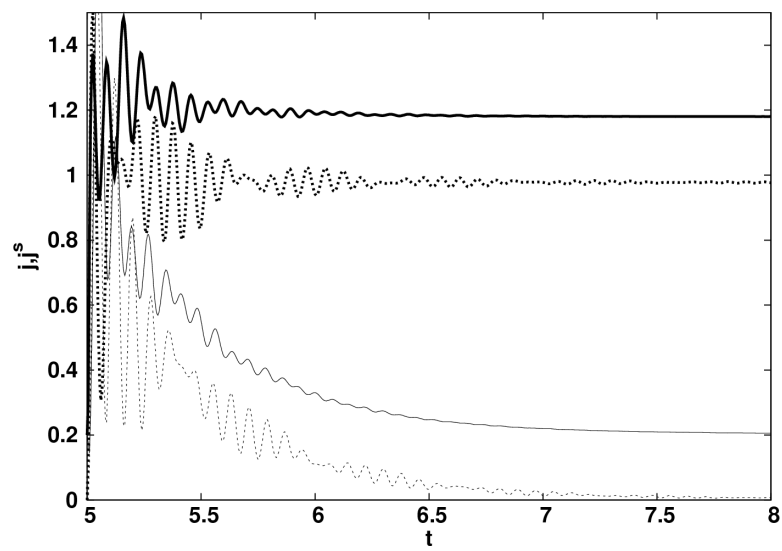

Fig. 1. The time dependence of the current $j_{\mathrm{L}}^{\downarrow}+j_{\mathrm{L}}^{\uparrow}$ (thick lines) and spin current $j_{\mathrm{L}}^{\uparrow}-j_{\mathrm{L}}^{\downarrow}$ (thin lines) flowing in the QD system in response to the sudden downshift of the QD energy level $(-80)$ and the right lead Fermi level $(-160)$. The solid (dotted) line corresponds to $U=15$ $(U=0)$ Coulomb interaction. $E_{\mathrm{Z}}=4$ and the step-like bias voltage pulse is supplied at $t=5$. The results for $U=0$ and $U=15$ are separated vertically by 0.2 for clarity.

In Fig. 2 we show the polarization ratio $r(t)=$ $2\left(j_{\text {tot }} j_{\text {spin }}\right) /\left(j_{\text {tot }}^{2}+j_{\text {spin }}^{2}\right)$, where $j_{\text {tot }}=j^{\uparrow}+j^{\downarrow}$ and $j_{\text {spin }}=$ $j^{\downarrow}-j^{\uparrow}$, respectively. The $r(t)$ is equal to \pm 1 for fully polarized currents and $r=0$ for pure charge or spin current [8]. We show $r(t)$ obtained for the step-like pulsed bias voltage with the period $T=1$ for vanishing and non-zero Coulomb interaction $U$. The thin lines correspond to the step-like bias pulse. We observe that in the case of the single bias pulse the polarization ratio strongly oscillates during the initial transient and is small otherwise, especially for the $U \neq 0$ case. Let us note that the quantum beats which are present on the spin current curves for vanishing Coulomb interaction (see Fig. 2) remain also very distinct on the $r(t)$ curves. The structure of $r(t)$ for the pulsed bias is quite different in comparison with the one-step bias pulse. Now we can observe the repeated time intervals in which the large values of the polarization ratio are present. However, the overall structure of $r(t)$ is different for the $U=0$ and $U \neq 0$ cases. The difference is especially remarkable in the second half-part of the period $T(5.5<t<6,6.5<t<7, \ldots)$. For the vanishing $U$ case we have rather a small polarization ratio corresponding to almost pure charge or spin current. On the contrary, for $U \neq 0$, we observe rather almost fully polarized currents.
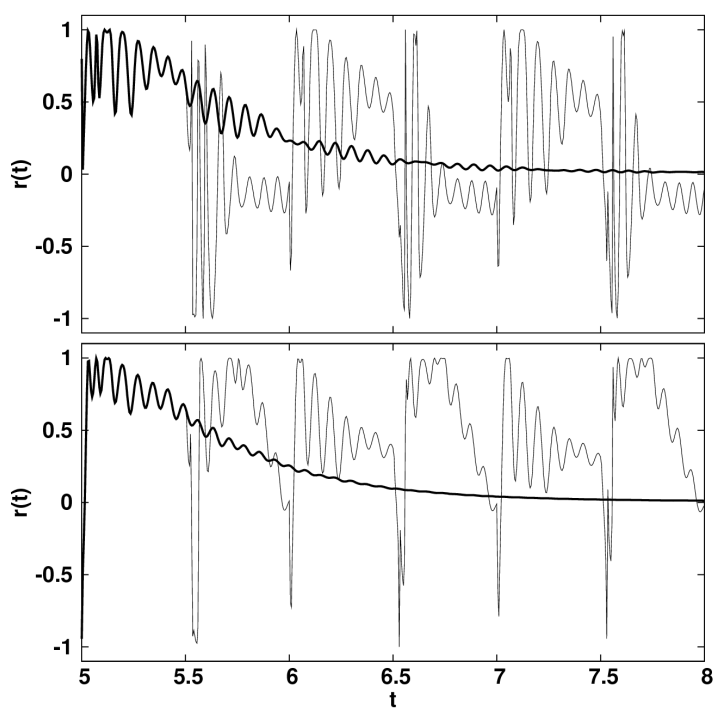

Fig. 2. The time dependence of the polarization ratio of the total current flowing in the QD system in response to the step-like pulsed bias voltage of the period $T=1$ supplied at $t=5$. The upper (lower) part corresponds to the vanishing $U, U=0(U=15)$. The thick line describes the case of the single step-like bias voltage pulse. The other parameters as in Fig. 1.

\section{Conclusion}

In conclusion, we have performed calculations of the transient tunneling current and QD occupancies in response to the step-like bias modulation. The influence of the Coulomb interaction between the electrons of the opposite spins localized in QD on the total current, spin current and spin polarization of the total current was analyzed. We showed that the coherent quantum beats in both the total and spin currents found in the case 
of vanishing Coulomb interaction are suppressed for the $U \neq 0$ case. We have also shown that applying the pulsed bias voltage one can obtain the positive, as well as the negative polarization ratios. For the non-zero Coulomb interaction almost fully polarized current occurs in some time windows as opposed to the almost pure charge or spin current for $U=0$.

\section{Acknowledgments}

The work of P.P. was supported by the Ministry of Science and Higher Education by grant No. N N202 109036 and work of R.T. was supported partially by grant No. N N202 187833

\section{References}

[1] A.-P. Jauho, N.S. Wingreen, Y. Meir, Phys. Rev. B 50, 5528 (1994).
[2] R. Aguado, G. Platero, Phys. Rep. 395, 1 (2004).

[3] R. Taranko, T. Kwapinski, E. Taranko, Phys. Rev. B 69, 165306 (2004)

[4] T. Kwapinski, R. Taranko, E. Taranko, Phys. Rev. B 72, $125312(2005)$

[5] F.M. Souza, Phys. Rev. B 76, 205315 (2007).

[6] F.M. Souza, A.P. Jauho, J.C. Egues, Phys. Rev. B 78, 155303 (2008).

[7] G. Stefanucci, C.-O. Almbladh, Phys. Rev. B 69 195318 (2004).

[8] E. Perfetto, G. Stefanucci, M. Cini, Phys. Rev. B 78, 155301 (2008). 